\title{
The significance of PSA/IGF-1 ratio in differentiating benign prostate hyperplasia from prostate cancer
}

\author{
G. Koliakos ${ }^{\mathrm{a}, *}$, D. Chatzivasiliou ${ }^{\mathrm{b}}$, \\ Th. Dimopoulos ${ }^{\mathrm{b}}, \mathrm{V}$. Trachana ${ }^{\mathrm{a}}$, \\ K. Paschalidou ${ }^{\mathrm{a}}$, V. Galiamoutsas ${ }^{\mathrm{b}}$, A. Triantos ${ }^{\mathrm{a}}$, \\ G. Chitas ${ }^{\mathrm{b}}$, A. Dimopoulos ${ }^{\mathrm{b}}$ and G. Vlatsas ${ }^{\mathrm{a}}$ \\ ${ }^{a}$ Department of Biological Chemistry, Medical \\ School, Aristotle University of Thessaloniki, Greece \\ ${ }^{\mathrm{b}}$ Urology Clinic, Hospital "Panagia", Social Security \\ Foundation, Thessaloniki, Greece
}

Received 8 May 2000

Accepted 20 December 2000

\begin{abstract}
The importance of insulin-like growth factor 1 (IGF-1) in human serum for the early diagnosis of prostate cancer is controversial. The IGF-1/PSA ratio may improve the performance of prostate specific antigen (PSA) as a prostate cancer marker.

IGF-1, along with PSA and free PSA concentration, was measured in the serum of 34 patients with prostate cancer and in 131 patients with benign prostatic hyperplasia (BPH).

Although IGF-1 concentration did not significantly differ between the groups, PSA/IGF-1 ratio could clearly distinguish the two groups. In patients with cancer but not in patients with BPH, IGF-1 concentration correlated with PSA and free PSA. The values of PSA and free PSA correlated with each other for both groups. Receivers Operating Curve (ROC) analysis indicated a better sensitivity to specificity ratio for PSA/IGF-1 than for PSA or Free/Total (F/T) PSA.
\end{abstract}

Keywords: PSA, IGF-1, prostate cancer, prostatic hypertrophy

\section{Introduction}

Prostate specific antigen (PSA) concentration in serum, is presently the most effective biochemical

*Correspondence to: George Koliakos, PO Box 17034, 54210, Thessaloniki, Greece. Tel.: +30 31 999123; Fax: +30 31 999004; E-mail: koliakos@med.auth.gr. disease marker for the diagnosis and monitoring of prostate cancer. However, PSA has been proved insufficient especially for the early detection of this malignancy. Thus, several concepts have been proposed attempting to optimize the clinical use of PSA measurements [6]. The introduction of free PSA and the ratio of free-to-total (F/T) PSA improved the diagnostic potential of PSA measurements, in selected men with elevated total serum PSA levels [19], due to the fact that F/T PSA is decreased in prostate cancer and increased in benign prostate hyperplasia (BHP) [18]. A low percentage of free PSA (below 10\%) is a powerful predictor of prostate cancer in patients with an elevated total PSA (above $4 \mathrm{ng} / \mathrm{ml}$ ) even after two negative prostate sextant biopsies [15]. On the other hand, F/T PSA does not improve significantly the specificity for the detection of prostate cancer in screening trials [2]; thus the combination of total PSA and digital rectal examination (DRE) remains the standard procedure for this purpose $[13,16,18]$.

It has been reported that high levels of insulin-like growth factor one (IGF-1) in human serum can predict the risk of prostate cancer in man $[14,22]$. In contrast to these observations other researchers concluded that serum IGF-1 concentration is not a useful marker of prostate cancer $[5,7,12]$. However when the IGF1/PSA ratio was considered an improved performance for the detection of prostate cancer was reported [7].

In an attempt to further study if IGF-1 concentration in serum can be useful for differentiating prostate cancer from BPH, IGF-1 concentration was measured in the serum of patients with prostate cancer diagnosed by sextant biopsy and in patients with BPH along with PSA and free PSA concentrations.

\section{Patients and methods}

One hundred and seventy one consecutive patients that were examined in our urology clinic between the 
years 1995 and 1997 were included in the study. The patients selected had no history of prior urological examination and they were complaining of symptoms that suggested prostatic disease. Blood sample was drawn before any prostatic examination. Serum was aliquoted and kept in $-70^{\circ} \mathrm{C}$ for PSA, free PSA and IGF- 1 estimations. The patients were then examined by DRE. Patients where prostatitis was suspected, were excluded from further examination. Follow up of these patients, revealed a decline in PSA and regression of symptoms, confirming thus the diagnosis of prostatitis.

In cases where DRE raised suspicion of malignancy, a digital palpation guided transrectal biopsy followed. An automatic biopsy system using a 18 gauge needle was employed (Monopty Biopsy Instrument, Bard, Urological division).

In cases where DRE was negative a transrectal ultrasound study (TRUS) was performed using a ATC 9 Ultra-mark system equipped with a 5.0 MHz head. In cases where malignancy was suggested a TRUS guided transrectal biopsy followed.

In cases where TRUS was negative a sextant prostatic biopsy using the automatic biopsy system followed (Bard Urological, Corington, GA).

In the cases where no cancer was detected the patients were reexamined 3 years after initial examination and had no signs of cancer as indicated by PSA and DRE examination. These patients were BPH. Of course in situ carcinoma can not be excluded.

The concentration of PSA in serum was measured using a commercial immunoradiometric assay (PSAIRMACT, Cis Biointernational, Gif Sur Yvette Cedex, France). The assay uses the coated tube technique with two monoclonal antibodies that recognize specific epitopes on free PSA and on the PSA-a1 antichymothrypsin complex (PSA-ACT) but not the PSA-a2 microglobulin complex (PSA-a2M). The intra assay variation for normal levels was $\pm 2.5 \%$ and the between assay variation was $\pm 4.8 \%$. The sensitivity limit of the assay was $0.04 \mathrm{ng} / \mathrm{ml}$.

Free PSA serum concentration was estimated using a commercial immunoradiometric assay (PSAIRMACT, Cis Biointernational, Gif SurYvette Cedex, France). The assay uses the coated tube technique with two monoclonal antibodies that recognize specific epitopes on free PSA with less than $1 \%$ crossreactivity for PSA-ACT. The intra assay variation for normal levels was $\pm 1.9 \%$ and the interassay variation was $\pm 4.2 \%$. The sensitivity limit of the assay was $0.02 \mathrm{ng} / \mathrm{ml}$.

IGF-1 has been estimated by a non extraction commercial immunoradiometric assay (Biocode Sclessin,
Belgium). The assay uses the coated tube technique with two monoclonal antibodies that recognize specific epitopes on IGF-1 with less than $1 \%$ crossreactivity for insulin, growth hormone or IGF-II. The intra assay variation for normal levels was $\pm 4.1 \%$ and the interassay variation was $\pm 4.3 \%$. The sensitivity limit of the assay was $2 \mathrm{ng} / \mathrm{ml}$.

For statistical evaluation of the data the statistical package SPSS for Windows (V.8) was used. The clinical sensitivity and specificity of the tests has been calculated using the results of the biopsy as a "golden standard" [9]. Clinical sensitivity and specificity data for various reference limits have been presented on receivers operating curves (ROC).

\section{Results}

Among the 171 patients, 24 were found to have prostatitis and were excluded from the study. Thirty four patients had prostate cancer indicated by biopsy and 113 patients had BPH with no signs of prostate cancer on 3 year follow up. The age of the patients with cancer $(67 \pm 9)$ did not differ significantly from the age of patients with $\mathrm{BPH}(69 \pm 7.4 P=0.363)$.

Among the patients finally selected, the concentration of IGF-1 did not significantly differ between patients with cancer and patients with $\mathrm{BPH}$; although a significant difference was found in the levels of PSA as well as free PSA between these two groups (Table 1).

Free/Total PSA ratio as well as PSA/IGF-1 ratio differed significantly between patients with cancer and patients with BPH (Table 1). In Tables 2 and 3, statistical correlation between the measured values is presented.

In patients with cancer, IGF-1 concentration did correlate with PSA and free PSA levels in serum (Table 2). On the other hand, no correlation between PSA or free PSA and IGF-1 was established for the patients with BPH (Table 3). The values of PSA and free PSA did correlate with each other for both groups. Free/Total PSA values correlated well with PSA/IGF-1 values for patients with cancer and, at a less significant level, for patients with BPH.

Finally, Receivers Operating Curve (ROC) analysis indicated that PSA/IGF-1 ratio offers a better sensitivity to specificity ratio than PSA or F/T PSA ratio (Fig. 1).

\section{Discussion}

The diagnostic potential of PSA measurements is limited mainly due to the increase in PSA concentra- 
Table 1

Comparison of the measured values between patients with prostate cancer ( $\mathrm{PCa})$ and patients with Benign Prostate Hyperplasia (BPH)

\begin{tabular}{|c|c|c|c|c|c|}
\hline & \multicolumn{2}{|c|}{$\mathrm{CaP}$} & \multicolumn{2}{|c|}{$\mathrm{BPH}$} & \multirow{2}{*}{$\begin{array}{l}\text { Level of significance } \\
(\mathrm{P})^{*}\end{array}$} \\
\hline & $x$ & SD & $x$ & $\mathrm{SD}$ & \\
\hline PSA & 8.7 & 11.5 & 2.4 & 2.3 & 0.007 \\
\hline Free PSA & 1.9 & 2.8 & 0.64 & 0.59 & 0.028 \\
\hline F/T PSA & 19.0 & 8.3 & 26.2 & 8.2 & 0.009 \\
\hline IGF-1 & 116.3 & 67.8 & 104.8 & 62.3 & 0.579 \\
\hline PSA/IGF-1 & 0.218 & 0.388 & 0.025 & 0.023 & 0.025 \\
\hline
\end{tabular}

${ }^{*}$ Student t-test.

Table 2

Significance of correlation $(\mathrm{P})$ between the measured values as calculated by Spearman correlation test (patients with prostate cancer)

\begin{tabular}{lccc}
\hline & Free PSA & IGF-1 & PSA/IGF-1 \\
\hline PSA & $<0.001$ & 0.066 & 0.006 \\
Free PSA & - & 0.022 & 0.003 \\
IGF-1 & 0.022 & - & $<0.001$ \\
F/T PSA & $<0.001$ & 0.050 & 0.002 \\
\hline
\end{tabular}

Table 3

Significance of correlation $(\mathrm{P})$ between the measured values as calculated by Spearman correlation test (patients with $\mathrm{BPH}$ )

\begin{tabular}{lccc}
\hline & Free PSA & IGF-1 & PSA/IGF-1 \\
\hline PSA & $<0.001$ & 0.111 & 0.002 \\
Free PSA & - & 0.361 & 0.763 \\
IGF-1 & 0.361 & - & 0.030 \\
F/T PSA & 0.009 & 0.595 & 0.042 \\
\hline
\end{tabular}

tions in benign prostatic hyperplasia (BPH) that is frequent in elderly people. The measurement of free PSA in serum improves the specificity of prostate cancer screening in selected men with elevated total serum PSA levels [19]. The F/T PSA ratio can also be used for the earlier prediction of a subsequent diagnosis of prostate cancer [17]. Increased IGF-1 concentration in serum is also related to increased risk of prostate cancer [14,22]. On the contrary, other researchers concluded that serum IGF-1 concentration is not a useful marker of prostate cancer $[5,7,12]$. However, when the IGF-1/PSA ratio was considered an improved performance for the detection of prostate cancer was reported [7].

In the present study the serum concentration of PSA, free PSA, IGF-1 as well as the ratios F/T PSA and PSA/IGF-1 were compared between patients with cancer, diagnosed for the first time and confirmed by biopsy and patients with $\mathrm{BPH}$ and no signs of cancer.

In agreement with other researchers [5,7,12], IGF-1 serum concentration, although slightly increased in patients with cancer, did not differentiate the two groups. However a significant difference in the PSA/IGF-1 ratio between the two groups was found. This result is in agreement with previous observations [7] and en- hances the possibilities of clinical use of this ratio to differentiate BPH from prostate cancer. Furthermore, as the data of the present study indicate, the PSA/IGF-1 ratio offers additional clinical value to total PSA measurement with a clinical sensitivity to specificity relationship (ROC curve) better than the corresponding F/T PSA curve.

IGF-1 is an active mediator of growth hormone (GH) involved in normal prostate development and growth [20]. Chronic excess of growth hormone (GH) and IGF-1 cause prostate overgrowth but not prostate cancer [4]. On the other hand several studies suggest that insulin like growth factors play an important role in the development and progression of cancer [23]. IGF-1 mRNA levels and IGF-1 concentration in serum are increased during prostate cancer progression in mice [11]. However it has been reported that IGF-1 serum concentrations are mainly related to prostate growth, being a marker for BPH and not for prostate cancer [5,7,12]. In contrast, other researchers conclude that there is no relationship between IGF-1 serum concentration and BPH [14].

A significant statistical correlation between free and total PSA has been observed in the present study. This correlation was expected since the equilibrium between free and bound molecule is a function of the concentration of the molecule and the binding proteins [8]. On the other hand the concentration of IGF-1 correlated with PSA and free PSA concentration only in patients with cancer but not in patients with BPH. The correlation between these two molecules in prostate cancer may reflect tumor growth and development [11]. On the other hand it seems that in BPH these two indices are not related to each other.

The main source of PSA is prostate, thus PSA in serum is increased in any prostate pathology [1]. IGF-1 is a major growth regulating molecule normally produced by liver as well as by other cell types, such as normal prostate cells under the influence of growth hormone [10]; however the molecule is overproduced by 
cancer cells [21]. IGF-1 is normally found in the seminal fluid together with PSA [3]. A slight increase in IGF-1 due to overproduction by prostate cancer cells or increased permeability from the seminal fluid may not significantly influence the total blood pool of IGF- 1 . Thus, as the data of the present study suggest, IGF-1 concentration in serum may be indicative for prostate cancer only if related to a prostate specific molecule such as PSA and cannot be used as an independent prostate cancer index.

\section{References}

[1] R.J. Ablin, A retrospective and prospective overview of prostate-specific antigen, J Canc Res Clin Oncol 123 (1997), 583-594.

[2] C.H. Bangma, R. Kranse, B.G. Blijenberg and F.H. Schröder, The value of screening tests in the detection of prostate cancer. Part I: results of a retrospective evaluation of 1726 men, Urology 46 (1995), 773-778.

[3] P. Cohen, D.M. Peehl and R.G. Rosenfeld, The IGF axis in the prostate, Horm Metab Res 26(2) (1994), 81-84.

[4] A. Colao, P. Marzullo, S. Spiezia, D. Ferone, A. Giaccio, G. Cerbone, R. Pivonello, C. Di Somma and G. Lombardi, Effect of growth hormone $(\mathrm{GH})$ and insulin-like growth factor I on prostate diseases: an ultrasonographic and endocrine study in acromegaly, GH deficiency, and healthy subjects, J Clin Endocrinol Metab 84(6) (1999), 1986-1991.

[5] C.W. Cutting, C. Hunt, J.A. Nisbet, J.M. Bland, A.G. Dalgleish and R.S. Kirby, Serum insulin-like growth factor-I is not a useful marker of prostate cancer, BJU Int 83(9) (1999), 996999.

[6] R. Daher and M. Beaini, Prostate-Specific Antigen and new related markers for prostate cancer, Clin Chem Lab Med 36(9) (1998), 671-681.

[7] B. Djavan, B. Bursa, C. Seitz, G. Soeregi, M. Remzi, A. Basharkhah, R. Wolfram and M. Marberger, Insulin-like growth factor 1 (IGF-1), IGF-1 density, and IGF-1/PSA ratio for prostate cancer detection, Urology 54(4) (1999), 603-606.

[8] D.M. Friefelder, Physical Biochemistry: Applications to Biochemistry and Molecular Biology, W.H. Freeman \& Co, New York, 1982.

[9] R.S. Galen, Beyond normality: the predictive value and effciency of medical diagnoses, John Wiley, New York, 1975.

[10] J.I. Jones and D.R. Clemmons, Insulin-like growth factors and their binding proteins: biological actions, Endocr Rev 16 (1995), 3-34.

[11] P.J. Kaplan, S. Mohan, P. Cohen, B.A. Foster and N.M. Green- berg, The insulin-like growth factor axis and prostate cancer: lessons from the transgenic adenocarcinoma of mouse prostate (TRAMP) model, Cancer Res 59(9) (1999), 2203-2209.

[12] R. Kurek, U.W. Tunn, O. Eckart, G. Aumuller, J. Wong and H. Renneberg, The significance of serum levels of insulin-like growth factor- 1 in patients with prostate cancer, BJU Int $\mathbf{8 5}$ (1) (2000), 125-129.

[13] P.J. Littrup, Future benefits and cost effectiveness of prostate cancer screening, American Cancer Society, Cancer 80 (1997), 1864-1870.

[14] C.S. Mantzoros, A. Tzonou, L.B. Signorello, M. Stampfer, D. Trichopoulos and H.O. Adami, Insulin-like growth factor 1 in relation to prostate cancer and benign prostatic hyperplasia, Br J Cancer 76 (1997), 1115-1118.

[15] T.O. Morgan, D.G. Mclead, E.S. Leifer, G.P. Murphy and J.W. Moul, Prospective use of free prostate-specific antigen to avoid repeat prostate biopsies in men with elevated total prostate-specific antigen, Urology 48(6A) (1996), 76-80.

[16] R.G. Nixon and M.K. Brawer, Enhancing the specificity of prostate-specific antigen (PSA): an overview of PSA density, velocity and age-specific reference ranges, Br J Urol 1 (1997), 61-67.

[17] J.D. Pearson, A.A. Luderer, E.J. Metter, A.W. Partin, D.W. Chan, J.L. Fozard and H.B. Carter, Longitudinal analysis of serial measurements of free and total PSA among men with and without prostatic cancer, Urology 48(6A) (1996), 4-9.

[18] K. Pettersson, T. Piironen, M. Seppala, L. Liukkonen, A. Christensson, M.T. Matikainen, M. Suonpaa, T. Lovgren and H. Lilja, Free and complexed propstate-specific antigen (PSA): in vitro stability, epitope map, and development of immunofluorometric assays for specific and sensitive detection of free PSA and PSA-a1-antichymotrypsin complex, Clin Chem $\mathbf{4 1}$ (1995), 1480-1488.

[19] A. Reissigl, H. Klocker, J. Pointner, K. Fink, W. Horninger, O. Ennemoser, H. Strasser, K. Colleselli, L. Holtl and G. Bartsch, Usefulness of the ratio free/total prostate-specific antigen in addition to total PSA levels in prostate cancer screening, Urology 48(6A) (1996), 62-66.

[20] W. Ruan, L. Powell-Braxton, J.J. Kopchick and D.L. Kleinberg, Evidence that insulin-like growth factor 1 and growth hormone are required for prostate gland development, Endocrinology 140(5) (1999), 1984-1989.

[21] M.B. Sporn and A.B. Roberts, Autocrine growth factors and cancer, Nature 313 (1985), 745-747.

[22] A. Wolk, C.S. Mantzoros, S.O. Andersson, R. Bergstrom, L.B. Signorello, P. Lagiou, H.O. Adami and D. Trichopoulos, Insulin-like growth factor 1 and prostate cancer risk: a population-based, case-control study, J Natl Cancer Inst 90(12) (1998), 911-915.

[23] H. Yu and H. Berkel, Insulin-like growth factors and cancer, $J$ La State Med Soc 151(4) (1999), 218-223. 


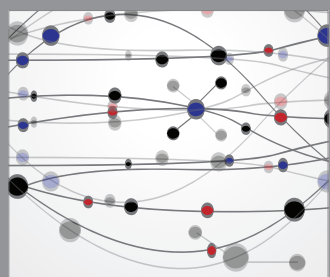

The Scientific World Journal
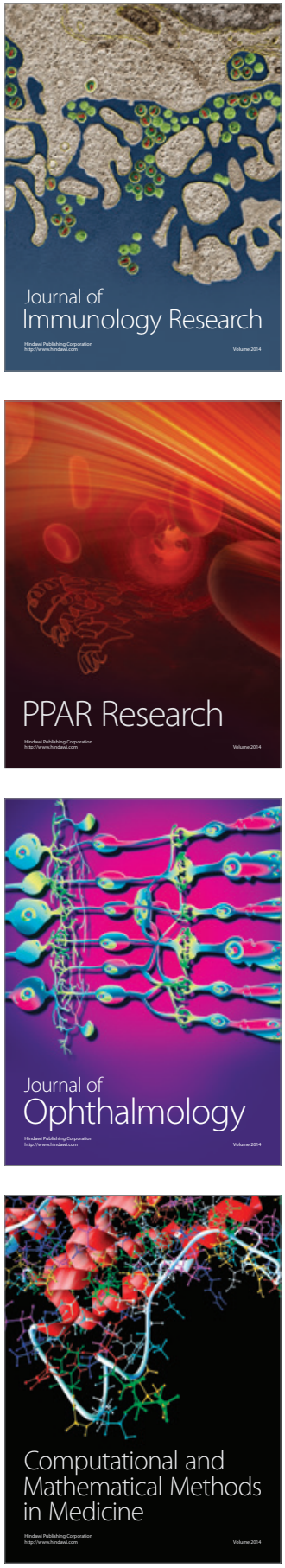

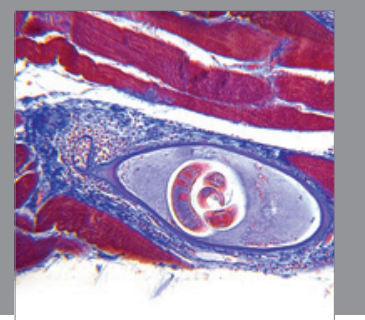

Gastroenterology

Research and Practice
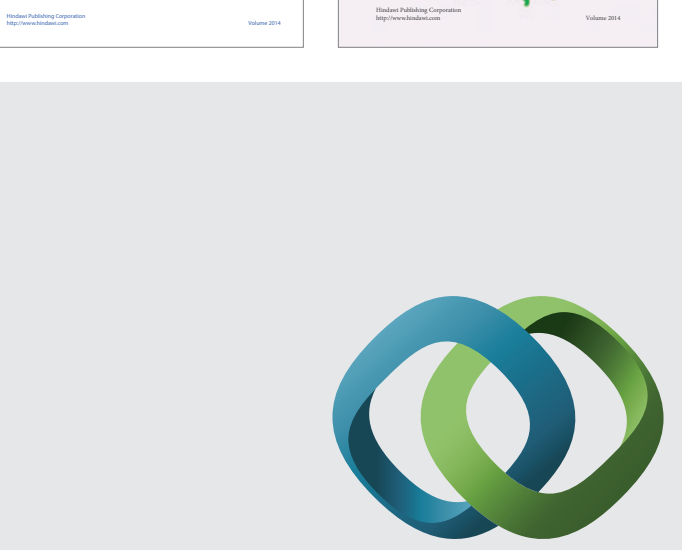

\section{Hindawi}

Submit your manuscripts at

http://www.hindawi.com
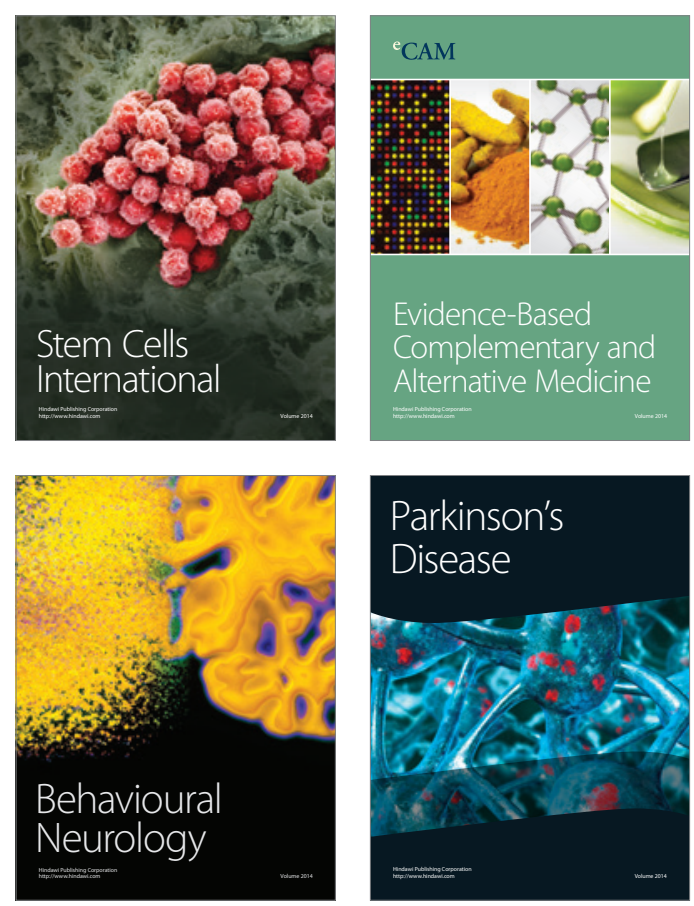

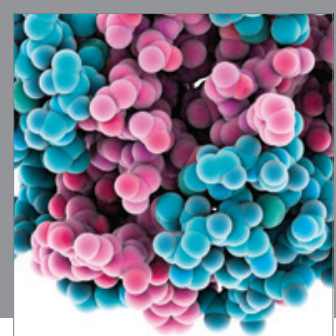

Journal of
Diabetes Research

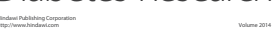

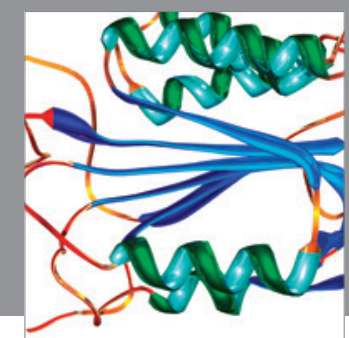

Disease Markers
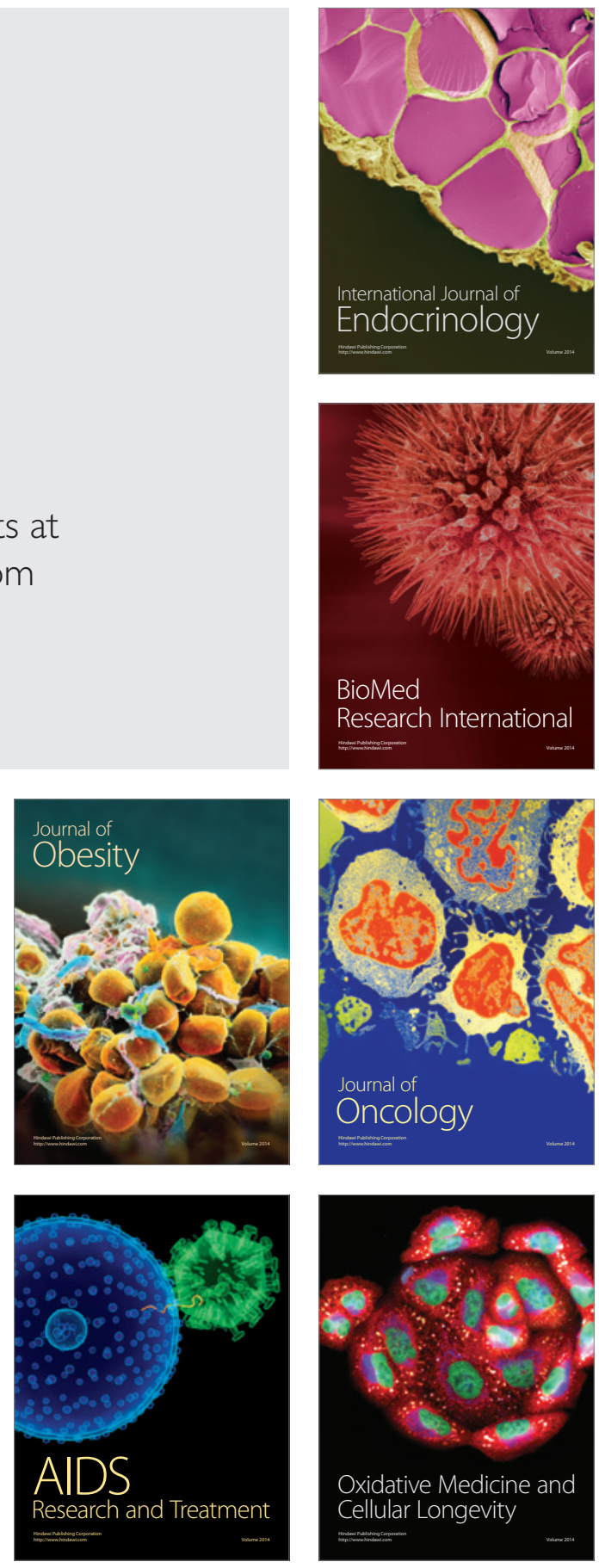